\title{
Participant No.
}

\section{Background Questionnaire}

1. How old are you?

2. What is your ethnicity? (Please circle one)
African
Chinese
Hispanic
East Indian
Caucasian
Other (please specify)

3 . In which country were you born?

4. Have you ever lived in a country other than your place of birth? Please circle Y / N

If yes, please list:

Country

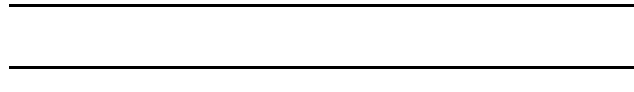

Length of time (approximately)

5. Where was your mother born?

6. What is her ethnicity?

7. Where was your father born?

8. What is his ethnicity?

9. Do you have any relatives (by birth or marriage) or close friends who are members of other ethnic or racial groups? Please circle Y / N

If yes, please list:

Their ethnicity

Relationship to you

How often you see them (circle one)
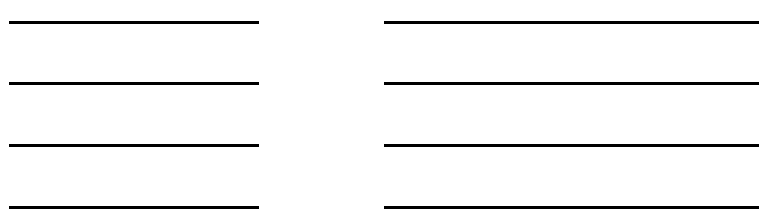

Daily Weekly Yearly <Yearly

Daily Weekly Yearly <Yearly

Daily Weekly Yearly <Yearly

Daily Weekly Yearly <Yearly

10. Have you ever lived with people from other ethnic groups? Please circle Y / N

If yes, please list:

Their ethnicity

Length of cohabitation

Your age when you moved in
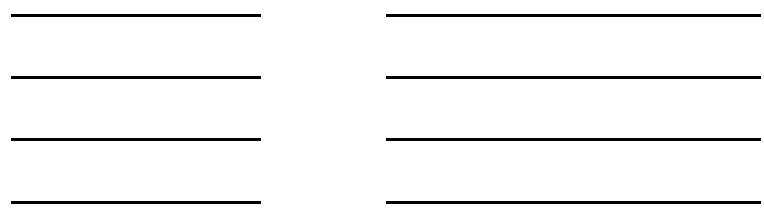

THANK YOU FOR YOUR PARTICIPATION! 